\title{
Estimating the impact of wide scale uptake of screening and medications for opioid use disorder in US prisons and jails
}

\author{
Alexandria Macmadu ${ }^{\mathrm{a}, \mathrm{b}}$, William C. Goedel ${ }^{\mathrm{a}}$, Joëlla W. Adams ${ }^{\mathrm{a}}$, Lauren Brinkley-Rubinstein ${ }^{\mathrm{c}, \mathrm{d}}$, \\ Traci C. Green ${ }^{\mathrm{a}, \mathrm{e}}$, Jennifer G. Clarke ${ }^{\mathrm{f}}$, Rosemarie A. Martin ${ }^{\mathrm{g}}$, Josiah D. Rich ${ }^{\mathrm{a}, \mathrm{b}}$, \\ Brandon D.L. Marshall ${ }^{\mathrm{a}, *}$ \\ ${ }^{a}$ Department of Epidemiology, Brown University School of Public Health, 121 South Main Street, Providence, RI, USA \\ ${ }^{\mathrm{b}}$ The Center for Prisoner Health and Human Rights, The Miriam Hospital, 8 Third Street, Providence, RI, USA \\ ${ }^{\mathrm{c}}$ Department of Social Medicine, University of North Carolina at Chapel Hill, 333 South Columbia Street, Chapel Hill, NC, 27516, USA \\ ${ }^{\mathrm{d}}$ Center for Health Equity Research, University of North Carolina at Chapel Hill, 335 South Columbia Street, Chapel Hill, NC, 27514, USA \\ ${ }^{\mathrm{e}}$ Department of Emergency Medicine, Boston University Medical Center, 725 Albany Street, Boston, MA, 02118, USA \\ ${ }^{\mathrm{f}}$ Rhode Island Department of Corrections, 40 Howard Avenue, Cranston, RI, 02920, USA \\ ${ }^{\mathrm{g}}$ Department of Behavioral and Social Science, Brown University, 121 South Main Street, Providence, RI 02903, USA
}

A R T I C L E I N F O

\section{Keywords:}

Opioids

Overdose

Prison

Jail

Medications for opioid use disorder

\begin{abstract}
A B S T R A C T
Background: Medications for opioid use disorder (OUD) are the most effective treatment for OUD, but uptake of these life-saving medications has been extremely limited in US prisons and jail settings, and limited data are available to guide policy decisions. The objective of this study was to estimate the impact of screening and treatment with medications for OUD in US prisons and jails on post-release opioid-related mortality.

Methods: We used data from the National Center for Vital Statistics, the Bureau of Justice Statistics, and relevant literature to construct Monte Carlo simulations of a counterfactual scenario in which wide scale uptake of screening and treatment with medications for OUD occurred in US prisons and jails in 2016.

Results: Our model predicted that 1840 (95\% Simulation Interval [SI]: -2757 - 4959) lives would have been saved nationally if all persons who were clinically indicated had received medications for OUD while incarcerated. The model also predicted that approximately 4400 (95\% SI: 2675 - 5557) lives would have been saved nationally if all persons who were clinically indicated had received medications for OUD while incarcerated and were retained in treatment post-release. These estimates correspond to 668 (95\% SI: -1008 1812) and 1609 (95\% SI: 972 - 2037) lives saved per 10,000 persons incarcerated, respectively.

Conclusions: Prison and jail-based programs that comprehensively screen and provide treatment with medications for OUD have the potential to produce substantial reductions in opioid-related overdose deaths in a highrisk population; however, retention on treatment post-release is a key driver of population level impact.
\end{abstract}

\section{Introduction}

The epidemic of opioid overdose is a growing crisis in the US. In 2016, 42,249 Americans died of an opioid-related overdose, more than any prior year in recorded history (Seth et al., 2018). This evolving crisis has disproportionately affected persons with recent experiences of incarceration (Binswanger et al., 2013; Brehm Christensen et al., 2006; Bukten et al., 2017; Huang et al., 2011; Merrall et al., 2010; Winter et al., 2015). In the US, the two-week period following release from prison or jail is associated with a 129-fold increase in the risk of death due to overdose relative to the general population (Binswanger et al., 2007). While medications for opioid use disorder (OUD)_including methadone, buprenorphine, and depot naltrexone-are the most effective treatment, they are not routinely available in most US prison and jails. There have been recent calls to ensure access to treatment within prisons and jails from health advocates, scientists, policy makers, and legal experts. The National Academies of Sciences recently stated that withholding these medications is unethical (Leshner and Mancher, 2019), and the Law Enforcement Action Partnership, a collection of current and former elected sheriffs, prosecutors, and other law enforcement professionals, has publicly called for the provision of medications for OUD within correctional facilities (Law Enforcement Action Partnership (LEAP), 2019). While there is increased support for provision of medications for OUD within correctional settings, available

\footnotetext{
* Corresponding author.

E-mail address: brandon_marshall@brown.edu (B.D.L. Marshall).
} 
data in the US are currently limited and preliminary (Green et al., 2018).

In England, investigators documented a 31\% reduction in drug-related poisoning deaths post-release among those who received medications for OUD while incarcerated compared to those who were not (Marsden et al., 2017). In New South Wales, Australia, investigators documented a $74 \%$ reduction in accidental drug-induced deaths when comparing those who had received medications for OUD while incarcerated and retained in treatment post-release to those who had not (Degenhardt et al., 2014). In the present study, we sought to estimate the expected reduction in opioid-related overdose deaths if wide scale uptake of screening and medications for OUD had occurred in all US prisons and jails in 2016; we make the assumption that similar program effectiveness w ould be achieved and that reductions in opioid-related overdose mortality in the US would be similar to those observed in England and Australia. For this analysis, we developed a model using data from several national and international sources. Using data from England, we conservatively estimated of the number of lives that might be saved by provision of medications for OUD in prisons and jails alone, and using data from Australia, we calculate a more ambitious estimate of the number of lives that might be saved by availability of medications for OUD in prisons and jails and post-release retention in treatment.

\section{Material and methods}

We estimate the reduction in opioid-related overdose mortality in each state if wide scale uptake of screening and medications for OUD had occurred in all US prisons and jails in 2016. We produce estimates for two counterfactual scenarios: (1) all persons who are clinically indicated receive medications for OUD while incarcerated, and (2) all persons who are clinically indicated receive medications for OUD while incarcerated and are retained in treatment post-release. The steps below were used to generate estimates for each counterfactual scenario.

First, we used data from the National Center for Health Statistics database to determine the total number of opioid overdose deaths for each state in 2016. Opioid-related overdose deaths were defined as having the following International Classification of Diseases, 10th Revision (ICD-10) codes: opium (T40.0), heroin (T40.1), natural and semisynthetic opioids (T40.2), methadone (T40.3), synthetic opioids other than methadone (T40.4), or other and unspecified narcotics (T40.6) as a contributing cause. We then multiplied the total number of opioid-related overdose deaths in each state by the proportion expected to occur during the one year post-release period ( $f O R M_{\text {postrelease }}$ ). This proportion was derived from several empirical sources, including estimates from North Carolina, Pennsylvania, Washington, and Rhode Island, and ranged from 3\% to 25\% (Binswanger et al., 2013, 2007; Green et al., 2018; Pizzicato et al., 2018; Ranapurwala et al., 2018). To reflect the uncertainty in this model parameter, we performed a Monte Carlo simulation which drew values from a uniform distribution ranging from $3 \%$ to $25 \%$, as these values reflect the upper and lower bounds in published data. This simulation process was repeated 10,000 times for each state. The following equation describes this process:

$$
N_{i} O R M * f O R M_{\text {postrelease }}=N_{i} O R M_{\text {postrelease }}
$$

where $N_{i} O R M$ is the reported number of opioid overdose deaths within the state, $f O R M_{\text {postrelease }}$ is the proportion of deaths expected to occur during the post-release period, and $N_{i} O R M_{\text {postrelease }}$ is the resulting estimate for the number of deaths occurring during the post-release period within the state.

Second, we calculated the number of overdose deaths expected under a counterfactual scenario where all persons who are clinically indicated receive medications for OUD while incarcerated in 2016. We created a normal distribution representing the potential percent reduction in opioid overdose mortality attributable to provision of medications for OUD in correctional settings $\left(f M O U D_{\text {effect }}\right)$. This distribution was derived using data from England reporting a 31\% (standard deviation [SD]: 17.3) reduction in drug-related poisoning deaths postrelease when comparing those who received medications for OUD while incarcerated to those who had not. We implemented stochastic processes to select the percent reduction from the normal distribution using Monte Carlo simulations. This process was repeated 10,000 times for each state to yield the expected number of opioid-related overdose deaths among individuals within a year post-release, had wide scale uptake of screening and medications for OUD occurred in prisons and jails in 2016.

$$
N_{i} O R M_{\text {postrelease }} * f M O U D_{\text {effect }}=N_{i} O R M_{\text {postrelease with MOUD }}
$$

We used the difference between this estimate and the previously calculated estimate of the number of opioid-related overdose deaths post-release in which no medications for OUD programs were implemented (broadly reflecting real-world conditions in 2016) to estimate the number of lives saved attributable to wide scale uptake of screening and medications for OUD in prisons and jails, or averted mortality $\left(N_{i} O R M_{\text {averted }}\right)$ :

$N_{i} O R M_{\text {postrelease }}-N_{i} O R M_{\text {postrelease with MOUD }}=N_{i} O R M_{\text {averted }}$

A national estimate was derived by summing state-specific estimates.

To estimate the number of lives saved per 10,000 persons incarcerated, we divided the simulated number of lives saved in each state $\left(N_{i} O R M_{\text {averted }}\right)$ by the state-specific combined prison and jail population point prevalence values $\left(N_{i}\right.$ Incarcerated $)$, and multiplied these values by 10,000 to yield the number of lives saved per 10,000 persons incarcerated $\left(N_{i} O R M_{\text {averted per } 10 K}\right)$ :

$\left(\frac{N_{i} O R M_{\text {averted }}}{N_{i} \text { Incarcerated }}\right) * 10,000=N_{i} O R M_{\text {averted per } 10 \mathrm{~K}}$

The steps above were repeated using a second normal distribution representing the potential percent reduction in opioid overdose mortality attributable to provision of medications for OUD in correctional settings and retention on treatment post-release $\left(f M O U D_{\text {effect }}\right)$. This distribution was derived using data from Australia reporting a 74\% (SD: 6.5) reduction in accidental drug-induced deaths when comparing those who had received medications for OUD while incarcerated and were retained in treatment post-release to those who were not.

$\mathrm{R}$ Studio was used to conduct the Monte Carlo simulations and produce all maps. All mean estimates are reported using simulation intervals (SI) which accounts for stochastic processes by reporting the 95\% upper and lower limits of the simulated output. As a simulation study involving aggregate level data, this analysis did not require oversight from an institutional review board.

\section{Results}

The estimated number of lives saved and lives saved per 10,000 persons incarcerated among persons with recent incarceration if wide scale uptake of screening and medications for OUD had occurred in US prisons and jails, by state in 2016, and stratified by estimates with receiving treatment alone and receiving treatment with post-release retention are reported in Table 1 . Under the first scenario, the model predicts that if all persons who were clinically indicated had received medications for OUD while incarcerated in 2016, approximately 1840 (95\% SI: -2757 - 4959) lives would have been saved nationally. We also estimated that 668 (95\% SI: -1008 - 1812) lives would be saved per 10,000 persons incarcerated.

Under the second scenario, the model predicts that if all persons who were clinically indicated had received medications for OUD while incarcerated and were retained in treatment post-release in 2016, 4400 (95\% SI: 2675 - 5557) lives would have been saved nationally, and 1609 (95\% SI: 972 - 2037) lives would be saved per 10,000 persons 
Table 1

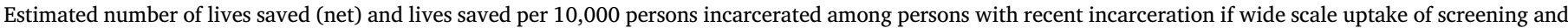

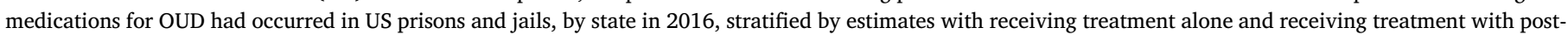
release retention.

\begin{tabular}{|c|c|c|c|c|}
\hline State & $\begin{array}{l}\text { Lives saved with } \\
\text { medications for OUD in } \\
\text { prisons \& jails } \\
\text { median }(95 \% \text { SI) }\end{array}$ & $\begin{array}{l}\text { Lives saved per } 10,000 \text { persons } \\
\text { incarcerated with medications for } \\
\text { OUD in prisons \& jails } \\
\text { median }(95 \% \mathrm{SI})\end{array}$ & $\begin{array}{l}\text { Lives saved with medications for } \\
\text { OUD in prisons \& jails and post- } \\
\text { release retention in treatment } \\
\text { median }(95 \% \mathrm{SI})\end{array}$ & $\begin{array}{l}\text { Lives saved per } 10,000 \text { persons incarcerated } \\
\text { with medications for OUD in prisons \& jails } \\
\text { and post-release retention in treatment } \\
\text { median }(95 \% \mathrm{SI})\end{array}$ \\
\hline Alabama & $15(-22-41)$ & $4(-5-10)$ & $35(21-45)$ & $9(5-11)$ \\
\hline Alaska & $4(-6-11)$ & $9(-14-25)$ & $10(6-12)$ & $23(14-27)$ \\
\hline Arizona & $34(-51-92)$ & $6(-9-17)$ & $80(49-101)$ & $15(9-18)$ \\
\hline Arkansas & $7(-11-20)$ & $3(-5-8)$ & $18(11-22)$ & $8(5-9)$ \\
\hline California & $89(-134-241)$ & $4(-7-12)$ & $212(129-270)$ & $10(6-13)$ \\
\hline Colorado & $23(-36-63)$ & $7(-11-20)$ & $56(34-70)$ & $17(11-22)$ \\
\hline Connecticut & $37(-55-102)$ & $25(-37-68)$ & $89(53-113)$ & $59(35-75)$ \\
\hline Delaware & $7(-11-18)$ & $11(-17-27)$ & $16(10-20)$ & $24(15-30)$ \\
\hline District of Columbia & $9(-13-25)$ & $50(-72-139)$ & $22(13-28)$ & $122(72-156)$ \\
\hline Florida & $121(-187-334)$ & $8(-12-22)$ & 289 (174-367) & $19(12-24)$ \\
\hline Georgia & $39(-61-109)$ & $4(-7-12)$ & $95(58-121)$ & $10(6-13)$ \\
\hline Hawaii & $3(-5-9)$ & $5(-9-16)$ & $8(5-10)$ & $14(9-18)$ \\
\hline Idaho & $5(-7-14)$ & $4(-6-12)$ & $12(7-16)$ & $11(6-14)$ \\
\hline Illinois & $87(-123-232)$ & $14(-20-38)$ & 204 (125-259) & $34(21-43)$ \\
\hline Indiana & $34(-52-95)$ & $8(-12-22)$ & $84(51-106)$ & $19(12-25)$ \\
\hline Iowa & $8(-12-22)$ & $6(-9-16)$ & $19(11-24)$ & $14(8-18)$ \\
\hline Kansas & $6(-10-17)$ & $3(-6-10)$ & $15(9-19)$ & $9(5-11)$ \\
\hline Kentucky & $43(-67-116)$ & $12(-19-33)$ & $103(62-131)$ & $30(18-38)$ \\
\hline Louisiana & $15(-23-41)$ & $3(-5-9)$ & $36(22-46)$ & $8(5-10)$ \\
\hline Maine & $13(-20-35)$ & $32(-49-85)$ & $32(19-40)$ & 78 (46-98) \\
\hline Maryland & $80(-121-216)$ & $28(-43-76)$ & $191(117-242)$ & $67(41-85)$ \\
\hline Massachusetts & $89(-126-237)$ & $46(-65-122)$ & $208(127-264)$ & $107(65-136)$ \\
\hline Michigan & $76(-115-208)$ & $13(-20-37)$ & $185(113-234)$ & $33(20-41)$ \\
\hline Minnesota & $17(-26-47)$ & $10(-16-29)$ & $41(25-52)$ & $25(15-32)$ \\
\hline Mississippi & $8(-12-21)$ & $3(-4-7)$ & $19(11-24)$ & $7(4-8)$ \\
\hline Missouri & $39(-62-108)$ & $9(-14-24)$ & $95(58-121)$ & 21 (13-27) \\
\hline Montana & $2(-3-5)$ & $4(-5-9)$ & $4(3-6)$ & $7(5-11)$ \\
\hline Nebraska & $2(-3-5)$ & $2(-3-6)$ & $5(3-6)$ & $6(3-7)$ \\
\hline Nevada & $17(-28-48)$ & $8(-14-24)$ & $42(26-54)$ & $21(13-27)$ \\
\hline New Hampshire & $19(-28-52)$ & $42(-62-116)$ & $46(28-58)$ & $102(62-129)$ \\
\hline New Jersey & $61(-93-166)$ & $19(-29-36)$ & 147 (91-187) & $46(28-58)$ \\
\hline New Mexico & $15(-23-41)$ & $10(-16-28)$ & $36(22-46)$ & $24(15-31)$ \\
\hline New York & $131(-200-358)$ & $18(-27-48)$ & $313(190-397)$ & $42(26-53)$ \\
\hline North Carolina & $66(-101-178)$ & $12(-19-33)$ & $156(94-198)$ & $29(17-37)$ \\
\hline North Dakota & $2(-4-6)$ & $6(-13-19)$ & $6(3-7)$ & $19(10-23)$ \\
\hline Ohio & $158(-239-426)$ & $22(-34-60)$ & $374(230-477)$ & $53(32-63)$ \\
\hline Oklahoma & $19(-31-52)$ & $5(-8-13)$ & $46(28-58)$ & $12(7-15)$ \\
\hline Oregon & $14(-22-37)$ & $7(-11-18)$ & $33(20-42)$ & $16(10-20)$ \\
\hline Pennsylvania & $98(-147-265)$ & $12(-18-32)$ & $233(140-296)$ & $28(17-36)$ \\
\hline Rhode Island & $12(-19-33)$ & $39(-61-106)$ & $29(17-36)$ & $94(55-116)$ \\
\hline South Carolina & $27(-40-75)$ & $8(-3-23)$ & 65 (39-83) & $20(12-26)$ \\
\hline South Dakota & $2(-3-5)$ & $3(-5-9)$ & $4(3-6)$ & $7(5-10)$ \\
\hline Tennessee & $52(-79-140)$ & $11(-16-29)$ & $123(75-156)$ & $25(15-32)$ \\
\hline Texas & $60(-88-164)$ & $3(-4-8)$ & $144(90-183)$ & $7(4-8)$ \\
\hline Utah & $20(-31-55)$ & $17(-26-47)$ & $49(30-62)$ & $42(26-53)$ \\
\hline Vermont & $4(-7-12)$ & $24(-41-71)$ & $10(6-13)$ & $59(35-76)$ \\
\hline Virginia & $49(-74-134)$ & $9(-13-23)$ & $117(70-148)$ & $20(12-26)$ \\
\hline Washington & $30(-47-83)$ & $10(-15-27)$ & $74(45-93)$ & $24(15-31)$ \\
\hline West Virginia & $32(-48-86)$ & $32(-48-85)$ & $75(45-96)$ & 74 (45-95) \\
\hline Wisconsin & $38(-58-103)$ & $11(-16-29)$ & $90(54-115)$ & $25(15-32)$ \\
\hline Wyoming & $2(-3-6)$ & $5(-8-15)$ & $5(3-7)$ & $13(8-18)$ \\
\hline Overall & $1840(-2757-4959)$ & $668(-1008-1812)$ & $4400(2675-5557)$ & 1609 (972-2037) \\
\hline
\end{tabular}

incarcerated. The estimated number of lives saved and per 10,000 persons incarcerated if wide scale uptake of screening and medications for OUD had occurred in US prisons and jails, by state in 2016, and stratified by estimates with receiving treatment alone (panels A and B) and receiving treatment with post-release retention (panels $\mathrm{C}$ and $\mathrm{D}$ ) are presented graphically in Fig. 1.

\section{Discussion}

Our model projected that if wide scale uptake of screening and medications for OUD had occurred in US prisons and jails in 2016, an estimated 1840 lives would have been saved by provision of medications for OUD in prisons and jails alone, and 4400 lives would have been saved by provision of medications for OUD in prisons and jails and programs to ensure post-release retention in treatment. For perspective, in a single year, the number of deaths that might be averted by provision of medications for OUD in prisons and jails with post-release retention in treatment is estimated to be greater than the number of lives lost in the War in Afghanistan (2216 lives), in Pearl Harbor (2403 lives), and in the terrorist attacks on 9/11 (2996 lives). Our estimates also represent approximately $4-10 \%$ of all opioid overdose deaths in 2016 (Scholl et al., 2019). By comparison, prescription drug monitoring programs were expected to prevent 600 overdose deaths in 2016-or $1.4 \%$ of all opioid overdose deaths in the same year (Patrick et al., 2016).

The current model examined the impact of screening and 
$\begin{array}{llll}\text { (A) Lives Saved } \quad 40 \quad 80 & 120\end{array}$
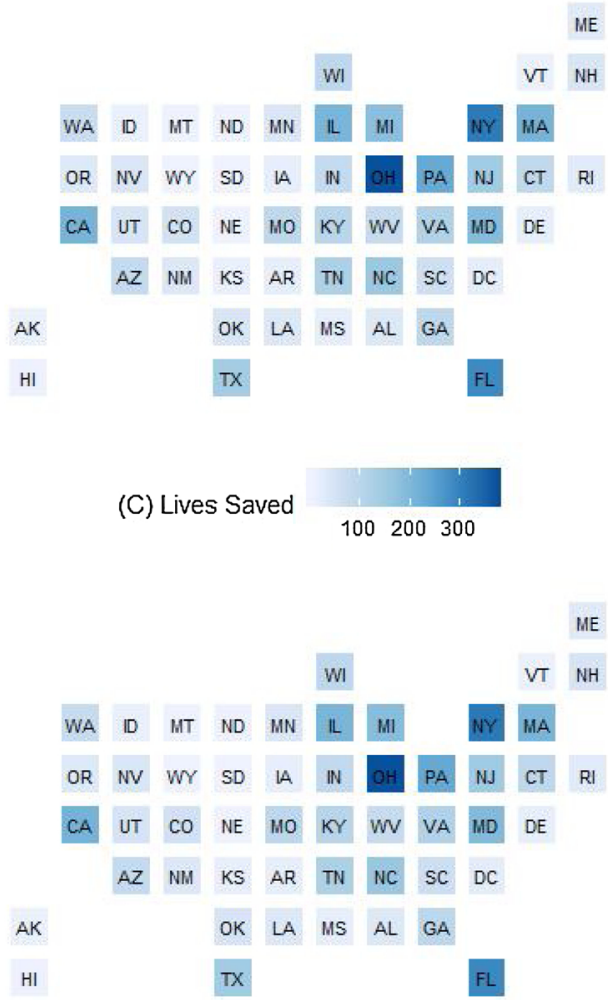

(B) Lives Saved per 10,000 Persons Incarcerated

$1020 \quad 3040 \quad 50$

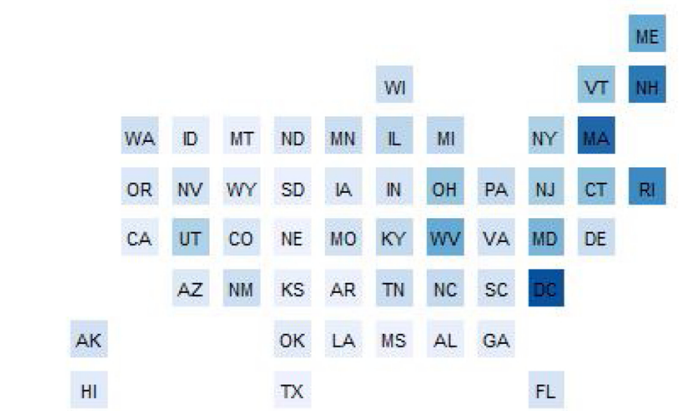

(D) Lives Saved per 10,000 Persons Incarcerated

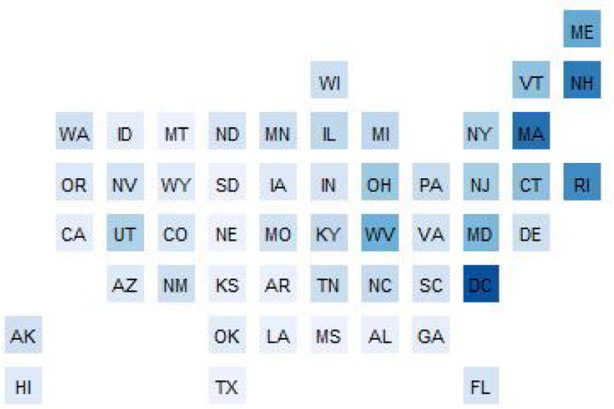

Fig. 1. Estimated number of lives saved and per 10,000 persons incarcerated if wide scale uptake of screening and medications for OUD had occurred in US prisons and jails, by state in 2016, stratified by estimates with receiving treatment alone (panels A and B) and receiving treatment with post-release retention (panels C and D).

medications for OUD programs on mortality alone. Additional benefits to expanding access to medications for OUD in correctional settings have been documented, including post-release reductions in non-medical opioid use, nonfatal overdose risk, and criminal behavior (BrinkleyRubinstein et al., 2018; Lee et al., 2016; Rich et al., 2015; Sharma et al., 2016), as well as improved perceptions of prison and jail safety and facility environment (Brinkley-Rubinstein et al., 2019). Undertreating OUD in correctional settings also has complex socioeconomic implications, including higher post-release healthcare costs, criminal justice costs, and overall community burden (Kinner and Wang, 2014). In addition to the number of human lives that might be preserved, jurisdictions might consider these supplementary benefits in weighing their decision to expand access to medications for OUD.

Current findings underscore the importance of post-release retention in treatment among persons who receive medications for OUD while incarcerated, as well as the need for concurrent interventions that mitigate overdose risk for this population. Naloxone distribution to individuals at release from incarceration (Bird et al., 2016) and to facility visitors (Huxley-Reicher et al., 2018) have demonstrated promise in reducing overdose mortality. Critically, pre-arrest diversion to community-based medications for OUD programs produces favorable outcomes for persons with OUD-in the absence of the myriad of harms that are associated with incarceration (Freudenberg and Heller, 2016).

Several limitations should be noted. First, our estimated rate of reductions in opioid-related mortality are derived from studies in England and Australia; these settings may differ from the US in critical ways (e.g., treatment capacity, healthcare access, medication treatments available) that may lead to increased or decreased program effects in different settings. Second, our estimates rely on opioid mortality data reported by the National Center for Health Statistics. These values are known to underestimate the true number of opioid-related deaths, as toxicological laboratory tests and the circumstances under which these tests are performed can vary by jurisdiction (Rudd, 2016); therefore, the estimates presented may be conservative. Third, limited data are available to estimate the proportion of opioid-related overdose deaths among individuals with prior-year incarceration. The data used to inform our input parameter were derived from studies that examined mortality rates among persons released from US prison systems; however, the risk of opioid overdose death post-release may differ for individuals who are released from jails. Fourth, medications for OUD are currently available in a limited number of prisons and jails nationwide. Our model assumes that the 2016 "standard of care" in prisons and jails (i.e., no access to medications for OUD) is applied nationwide; therefore, our model may overestimate the number of lives that might be saved in those jurisdictions that did provide medications for OUD to individuals who are incarcerated during this year. However, this limitation is somewhat minor, as very few prisons and jails nationwide provided access to medications for OUD to persons who were incarcerated during that time.

\section{Conclusions}

Our model projected that wide scale uptake of screening and treatment with medications for OUD in prison and jail settings would produce substantial reductions in opioid-related overdose deaths. These findings can be used by lawmakers, state public health officials, and prison and jail administrators when considering investments and potential impacts of interventions that can reduce overdose for persons who are incarcerated and for their overall jurisdiction, particularly in those states identified as having the highest numbers of potential deaths 
averted. Jurisdictions that lack community capacity for medications for OUD should build and expand these resources in tandem with prison and jail-based provision of medications for OUD to ensure that postrelease retention in treatment can be attained.

\section{Contributors}

AM, BDLM, JDR, and WCG conceptualized the study and design. AM developed initial drafts of the manuscript. AM and WCG conducted all analyses. JWA contributed significantly to writing the methods section. WCG, JWA, LBR, TCG, JGC, RAM, JDR, and BDLM provided iterative and substantive feedback on the overall approach and all drafts of the manuscript. All authors have approved the final manuscript.

\section{Role of funding source}

The work of AM, JR, and BDLM was supported by grants from the National Institute on Drug Abuse (K24DA022112 and R21DA044443). Their work was also supported by the COBRE on Opioids and Overdose, which is funded by the National Institute of General Medical Sciences of the National Institutes of Health (P20GM125507). The content is solely the responsibility of the authors and does not necessarily represent the official views of the National Institutes of Health.

\section{Declaration of Competing Interest}

No conflicts declared.

\section{Appendix A . Supplementary data}

Supplementary material related to this article can be found, in the online version, at doi:https://doi.org/10.1016/j.drugalcdep.2020. 107858.

\section{References}

Binswanger, I.A., Stern, M.F., Deyo, R.A., Heagerty, P.J., Cheadle, A., Elmore, J.G. Koepsell, T.D., 2007. Release from prison-a high risk of death for former inmates. N. Engl. J. Med. 356 (2), 157-165.

Binswanger, I.A., Blatchford, P.J., Mueller, S.R., Stern, M.F., 2013. Mortality after prison release: opioid overdose and other causes of death, risk factors, and time trends from 1999 to 2009. Ann. Intern. Med. 159 (9), 592-600.

Bird, S.M., McAuley, A., Perry, S., Hunter, C., 2016. Effectiveness of Scotland's National Naloxone Programme for reducing opioid-related deaths: a before (2006-10) versus after (2011-13) comparison. Addiction 111 (5), 883-891.

Brehm Christensen, P., Hammerby, E., Smith, E., Bird, S.M., 2006. Mortality among Danish drug users released from prison. Int. J. Prison. Health 2 (1), 13-19.

Brinkley-Rubinstein, L., McKenzie, M., Macmadu, A., Larney, S., Zaller, N., Dauria, E., Rich, J., 2018. A randomized, open label trial of methadone continuation versus forced withdrawal in a combined US prison and jail: findings at 12 months postrelease. Drug Alcohol Depend. 184, 57-63.

Brinkley-Rubinstein, L., Peterson, M., Clarke, J., Macmadu, A., Truong, A., Pognon, K., Parker, M., Marshall, B.D., Green, T.C., Martin, R., Stein, L., Rich, J.D., 2019. The benefits and implementation challenges of the first state-wide comprehensive medication for addictions program in a unified jail and prison setting. Accepted to Drug Alcohol Depend.

Bukten, A., Stavseth, M.R., Skurtveit, S., Tverdal, A., Strang, J., Clausen, T., 2017. High risk of overdose death following release from prison: variations in mortality during a 15-year observation period. Addiction 112 (8), 1432-1439.

Degenhardt, L., Larney, S., Kimber, J., Gisev, N., Farrell, M., Dobbins, T., Weatherburn, D.J., Gibson, A., Mattick, R., Butler, T., 2014. The impact of opioid substitution therapy on mortality post-release from prison: retrospective data linkage study. Addiction 109 (8), 1306-1317.

Freudenberg, N., Heller, D., 2016. A review of opportunities to improve the health of people involved in the criminal justice system in the United States. Annu. Rev. Public Health 37, 313-333.

Green, T.C., Clarke, J., Brinkley-Rubinstein, L., Marshall, B.D., Alexander-Scott, N., Boss, R., Rich, J.D., 2018. Postincarceration fatal overdoses after implementing medications for addiction treatment in a statewide correctional system. JAMA Psychiatry 75 (4), 405-407.

Huang, Y.F., Kuo, H.S., Lew-Ting, C.Y., Tian, F., Yang, C.H., Tsai, T.I., Gange, S.J., Nelson, K.E., 2011. Mortality among a cohort of drug users after their release from prison: an evaluation of the effectiveness of a harm reduction program in Taiwan. Addiction 106 (8), 1437-1445.

Huxley-Reicher, Z., Maldjian, L., Winkelstein, E., Siegler, A., Paone, D., Tuazon, E., Nolan, M.L., Jordan, A., MacDonald, R., Kunins, H.V., 2018. Witnessed overdoses and naloxone use among visitors to Rikers Island jails trained in overdose rescue. Addict. Behav. 86, 73-78.

Kinner, S.A., Wang, E.A., 2014. The case for improving the health of ex-prisoners. Am. J. Public Health 104 (8), 1352-1355.

Law Enforcement Action Partnership (LEAP), 2019. Open Letter From Current and Former Elected Sheriffs, Elected Prosecutors, and Other Law Enforcement Professionals.

Lee, J.D., Friedmann, P.D., Kinlock, T.W., Nunes, E.V., Boney, T.Y., Hoskinson Jr., R.A., Wilson, D., McDonald, R., Rotrosen, J., Gourevitch, M.N., 2016. Extended-release naltrexone to prevent opioid relapse in criminal justice offenders. N. Engl. J. Med. 374 (13), 1232-1242.

Leshner, A., Mancher, M., 2019. Medications for opioid use disorder save lives. Nat. Acad. Sci.

Marsden, J., Stillwell, G., Jones, H., Cooper, A., Eastwood, B., Farrell, M., Lowden, T., Maddalena, N., Metcalfe, C., Shaw, J., 2017. Does exposure to opioid substitution treatment in prison reduce the risk of death after release? A national prospective observational study in England. Addiction 112 (8), 1408-1418.

Merrall, E.L., Kariminia, A., Binswanger, I.A., Hobbs, M.S., Farrell, M., Marsden, J., Hutchinson, S.J., Bird, S.M., 2010. Meta-analysis of drug-related deaths soon after release from prison. Addiction 105 (9), 1545-1554.

Patrick, S.W., Fry, C.E., Jones, T.F., Buntin, M.B., 2016. Implementation of prescription drug monitoring programs associated with reductions in opioid-related death rates. Health Aff. 35 (7), 1324-1332.

Pizzicato, L.N., Drake, R., Domer-Shank, R., Johnson, C.C., Viner, K.M., 2018. Beyond the walls: risk factors for overdose mortality following release from the Philadelphia Department of Prisons. Drug Alcohol Depend. 189, 108-115.

Ranapurwala, S.I., Shanahan, M.E., Alexandridis, A.A., Proescholdbell, S.K., Naumann, R.B., Edwards Jr., D., Marshall, S.W., 2018. Opioid overdose mortality among former North Carolina inmates: 2000-2015. Am. J. Public Health 108 (9), 1207-1213.

Rich, J.D., McKenzie, M., Larney, S., Wong, J.B., Tran, L., Clarke, J., Noska, A., Reddy, M., Zaller, N., 2015. Methadone continuation versus forced withdrawal on incarceration in a combined US prison and jail: a randomised, open-label trial. Lancet 386 (9991), 350-359.

Rudd, R.A., 2016. Increases in drug and opioid-involved overdose deaths-united States, 2010-2015. MMWR Morb. Mortal. Wkly. Rep. 65.

Scholl, L., Seth, P., Kariisa, M., Wilson, N., Baldwin, G., 2019. Drug and opioid-involved overdose deaths-united States, 2013-2017. Morb. Mortal. Wkly. Rep. 67 (5152), 1419 .

Seth, P., Scholl, L., Rudd, R.A., Bacon, S., 2018. Overdose deaths involving opioids, cocaine, and psychostimulants—united States, 2015-2016. Am. J. Transplant. 18 (6), 1556-1568.

Sharma, A., O'Grady, K.E., Kelly, S.M., Gryczynski, J., Mitchell, S.G., Schwartz, R.P. 2016. Pharmacotherapy for opioid dependence in jails and prisons: research review update and future directions. Subst. Abuse Rehabil. 7, 27.

Winter, R., Stoové, M., Degenhardt, L., Hellard, M., Spelman, T., Jenkinson, R., McCarthy, D., Kinner, S., 2015. Incidence and predictors of non-fatal drug overdose after release from prison among people who inject drugs in Queensland. Australia. Drug alcohol depend. 153, 43-49. 